\title{
Tutte polynomials of bracelets
}

\author{
Norman Biggs
}

Received: 15 May 2009 / Accepted: 15 February 2010 / Published online: 2 March 2010

(C) Springer Science+Business Media, LLC 2010

\begin{abstract}
The identity linking the Tutte polynomial with the Potts model on a graph implies the existence of a decomposition resembling that previously obtained for the chromatic polynomial. Specifically, let $\left\{G_{n}\right\}$ be a family of bracelets in which the base graph has $b$ vertices. It is shown here (Theorems 3 and 4) that the Tutte polynomial of $G_{n}$ can be written as a sum of terms, one for each partition $\pi$ of a nonnegative integer $\ell \leq b$ :

$$
(x-1) T\left(G_{n} ; x, y\right)=\sum_{\pi} m_{\pi}(x, y) \operatorname{tr}\left(N_{\pi}(x, y)\right)^{n} .
$$

The matrices $N_{\pi}(x, y)$ are (essentially) the constituents of a 'Potts transfer matrix', and a formula for their sizes is obtained. The multiplicities $m_{\pi}(x, y)$ are obtained by substituting $k=(x-1)(y-1)$ in the expressions $m_{\pi}(k)$ previously obtained in the chromatic case. As an illustration, explicit calculations are given for some small bracelets.
\end{abstract}

Keywords Tutte polynomial $\cdot$ Potts model $\cdot$ Transfer matrix $\cdot$ Specht modules

\section{Introduction}

The Tutte polynomial of a graph $G$ is a two-variable polynomial $T(G ; x, y)$ that provides a great deal of useful information about $G$. It has applications to the study of colourings and flows on $G$ [5], as well as reliability theory [13, 18], knot theory [20], and statistical physics $[6-8,12]$.

\footnotetext{
N. Biggs ( $₫)$

Department of Mathematics, London School of Economics, Houghton Street, London WC2A 2AE, UK

e-mail: n.1.biggs@1se.ac.uk
} 
A paper [4] published in 1972 discussed families of graphs $\left\{G_{n}\right\}$ for which the Tutte polynomials $T_{n}=T\left(G_{n} ; x, y\right)$ satisfy a linear recursion of the form

$$
T_{n+r}+a_{1} T_{n+r-1}+\cdots+a_{r} T_{n}=0
$$

where the $a_{i}$ are polynomials in $x$ and $y$ with integer coefficients. The families were of the kind that we now call bracelets (see Sect. 2 for the definitions). More examples of this kind were studied by D.A. Sands in his thesis [15], but work on these lines soon ground to a halt. This was due partly to the primitive state of computer algebra at the time and partly to the fact that the only theoretical method available was the deletion-contraction algorithm, which is very inefficient.

Around the same time Temperley and Lieb [19] showed that the Tutte polynomial is closely related to the 'interaction models' that occur in statistical physics and that tools from that field can be applied. One such tool is the transfer matrix, which had been used in physics as long ago as $1941[10,11]$. Much later it transpired that, in the special case of the chromatic polynomial, the transfer matrix could be analysed by applying the theory of representations of the symmetric group, and this led to explicit formulae for the chromatic polynomials of bracelets [2, 3].

In this paper similar methods will be applied to the Tutte polynomial. The key is the identity linking the Tutte polynomial with the Potts model on a graph, which implies the existence of a decomposition resembling that used in the chromatic case. Specifically, let $\left\{G_{n}\right\}$ be a family of bracelets in which the base graph has $b$ vertices. Then we shall show that the Tutte polynomial of $G_{n}$ can be written as a sum of terms, one for each partition $\pi$ of a nonnegative integer $\ell \leq b$ :

$$
(x-1) T\left(G_{n} ; x, y\right)=\sum_{\pi} m_{\pi}(x, y) \operatorname{tr}\left(N_{\pi}(x, y)\right)^{n} .
$$

The matrices $N_{\pi}(x, y)$ are (essentially) the constituents of a 'Potts transfer matrix' (Sect. 2), and their 'multiplicities' $m_{\pi}(x, y)$ are obtained by substituting $k=$ $(x-1)(y-1)$ in the expressions $m_{\pi}(k)$ previously obtained in the chromatic case (Sect. 3). As an illustration, we shall carry out the calculations for the Tutte polynomials of the cubic and quartic plane ladders.

\section{The Potts transfer matrix}

Let $G$ be a connected graph with vertex set $V$ and edge-set $E$, and let $[k]$ denote the set of integers $1,2, \ldots, k$. In the Potts model on $G$ each 'state' $\sigma: V \rightarrow[k]$ is assigned a weight $y^{m(\sigma)}$, where $m(\sigma)$ is the number of $\sigma$-monochromatic edges, that is, the number of edges $v w$ such that $\sigma(v)=\sigma(w)$. Since the elements of $[k]$ represent attributes, all of which have similar properties, the symmetric group of all permutations of $[k]$ acts on the set of states in the obvious way.

The partition function for the Potts model is defined to be

$$
Z P(G ; k, y)=\sum_{\sigma: V \rightarrow[k]} y^{m(\sigma)} .
$$


$Z P$ is a polynomial function of the two parameters $k$ and $y$. For example, suppose $G=K_{3}$, with $V=\{1,2,3\}$ and $E=\{12,13,23\}$. Then the weight is $y^{3}$ for $k$ states, $y$ for $3 k(k-1)$ states, and 1 for the remaining $k(k-1)(k-2)$ states. Hence

$$
Z P\left(K_{3} ; k, y\right)=k y^{3}+3 k(k-1) y+k(k-1)(k-2) .
$$

The result holds only for integer values of $k$, but we can consider $Z P$ as a polynomial in $\mathbb{Z}[k, y]$, given by the same formula. (Alternatively, a physicist might prefer to use the well-known expansion of $Z P$ in terms of subgraphs [9] in order to justify the extension to real or complex values of $k$.) Now it turns out that if we introduce a new indeterminate $x$ defined by the substitution $k=(x-1)(y-1)$, then $Z P(G ;(x-$ $1)(y-1), y)$ is divisible by $(x-1)(y-1)^{|V|}$ in $\mathbb{Z}[x, y]$. For example,

$$
\begin{aligned}
Z P & \left(K_{3} ;(x-1)(y-1), y\right) \\
= & (x-1)(y-1) y^{3}+3(x-1)(y-1)(x y-x-y) y \\
& +(x-1)(y-1)(x y-x-y)(x y-x-y-1) \\
= & (x-1)(y-1)^{3}\left(x^{2}+x+y\right) .
\end{aligned}
$$

This is a particular case of a general result, established by a calculation which also proves that the complementary factor is the Tutte polynomial $T(G ; x, y)$.

Theorem 1 The following identity holds in $\mathbb{Z}[x, y]$ :

$$
(x-1)(y-1)^{|V|} T(G ; x, y)=Z P(G ;(x-1)(y-1), y) .
$$

Proof This result is folklore from the 1970s, and may be found in [1, 19]; a recent and authoritative account is given by Sokal [16].

A bracelet $G_{n}$ is formed by taking $n$ copies of a graph $B$ and joining each copy to the next by a set of links $L$ (with $n+1=1$ by convention). For each choice of $B$ and $L$, we get a family of graphs for which the transfer matrix method is appropriate.

Let $V$ be the vertex-set of the base graph $B$. Construct the graph $J$ which has two disjoint copies of $V$ for its vertex-set, say $V_{1}$ and $V_{2}$, and the following edges. The vertices in $V_{1}$ are joined among themselves by edges as in $B$, the vertices in $V_{1}$ are joined to those in $V_{2}$ by the links $L$, and the vertices in $V_{2}$ are not joined among themselves. Suppose $J$ is 'coloured' by assigning to $V_{1}$ the state $\sigma$, and to $V_{2}$ the state $\tau$. Now we say that an edge of $J$ is $(\sigma, \tau)$-monochromatic if both its vertices receive the same colour.

The matrix $M=M(k, y)$ has $k^{|V|}$ rows and columns, indexed by the states $\sigma$ and $\tau$, and the entries of $M$ are given by

$$
M_{\sigma \tau}=y^{\mu(\sigma, \tau)}
$$

where $\mu(\sigma, \tau)$ is the number of $(\sigma, \tau)$-monochromatic edges, as defined above. 
Theorem 2 With the notation as above,

$$
Z P\left(G_{n} ; k, y\right)=\operatorname{tr} M(k, y)^{n} .
$$

Proof The proof is standard. A version directly applicable to the present formulation can be found in [1, p. 26].

The essence of the transfer matrix method is that the eigenvalues of $M^{n}$ are the $n$th powers of the eigenvalues of $M$, so the spectrum of $M$ determines $Z P\left(G_{n} ; k, y\right)$ for all values of $n$. Furthermore, $M$ commutes with the action of the symmetric group $\mathrm{Sym}_{k}$ on the states, and hence the spectral decomposition of $M$ can be deduced from the corresponding $\mathrm{Sym}_{k}$-module.

Chang and Shrock [6-8] have obtained significant results about the expansion of $Z P$ when the graphs $G_{n}$ are lattice graphs with various boundary conditions. In our terms, these graphs are bracelets in which $B$ is a path or cycle. When the linking set $L$ is a matching, the graphs are square lattices, and by augmenting the matching the triangular and hexagonal lattices can be obtained. If $B$ has two vertices, the square lattice graph is the graph we call the cubic plane ladder $\mathrm{CPL}_{n}$, and the triangular lattice graph is the quartic plane ladder $\mathrm{QPL}_{n}$. In the papers $[7,8]$ the method used by Chang and Shrock was to obtain the entries of the relevant transfer matrices by explicit calculations, using diagrammatic representations of the various possibilities for the states. By this means they calculated the Tutte polynomials of many kinds of lattice graphs, including $\mathrm{CPL}_{n}$ and $\mathrm{QPL}_{n}$ (see (4.17), (5.23), (8.1), (8.3) in [7]). The Chang-Shrock method could also be described in terms of bases for the constituents of the corresponding $\mathrm{Sym}_{k}$-modules, and in Sect. 4 we shall illustrate the application of our main theorem by calculating the Tutte polynomials of $\mathrm{CPL}_{n}$ and $\mathrm{QPL}_{n}$ in this way.

\section{The Specht decomposition}

In this section $b$ and $k$ are fixed positive integers, and $[b],[k]$ are sets of the corresponding sizes (for convenience, $k>2 b$ ).

Following the remarks in the previous section, we begin by considering certain modules determined by actions of the symmetric group. For a partition $\pi$ of an integer $\ell$, we write $|\pi|=\ell$ and denote the parts of $\pi$ by $\pi_{1}, \pi_{2}, \ldots, \pi_{\ell}$, where zeros are included as necessary. Let $d(\pi)$ be the dimension of the irreducible representation of the symmetric group $\operatorname{Sym}_{\ell}$ that corresponds to $\pi$. There is an explicit formula for $d(\pi)$, known as the hook formula [13, p. 124]. For $k>2|\pi|$, let $\pi^{k}$ denote the partition of $k$ formed from $\pi$ by adding a part $k-|\pi|$. The hook formula for $d\left(\pi^{k}\right)$ turns out to be a polynomial function of $k$ [3]: we write it as

$$
m_{\pi}(k)=\frac{d(\pi)}{|\pi| !} \prod_{i=1}^{|\pi|}\left(k-|\pi|-\pi_{i}+i\right) .
$$


For example,

$$
m_{[300]}(k)=\frac{1}{6} k(k-1)(k-5), \quad m_{[210]}(k)=\frac{1}{3}(k-2)(k-4)(k-6),
$$

We also need the conventional value $m_{o}(k)=1$, where $o$ is the partition of 0 with no parts.

Let $\iota:[b] \rightarrow[k]$ be an injection and consider the action $\iota \mapsto \alpha \iota$ of $\operatorname{Sym}_{k}$ on the vector space generated by such injections. In the chromatic case it was shown [3] that the resulting module has $e(b, \pi)$ constituents (Specht modules) of dimension $m_{\pi}(k)$, where

$$
e(b, \pi)=\left(\begin{array}{c}
b \\
|\pi|
\end{array}\right) d(\pi) .
$$

Let $S(b, r)$ denote the number of partitions of a set of size $b$ into $r$ parts (the Stirling number of the second kind).

Theorem 3 The module defined by the action of $\mathrm{Sym}_{k}$ on the vector space generated by all functions $[b] \rightarrow[k]$ has $e^{*}(b, \pi)$ constituents of dimension $m_{\pi}(k)$, where

$$
e^{*}(b, \pi)=\sum_{r=1}^{b} S(b, r)\left(\begin{array}{c}
r \\
|\pi|
\end{array}\right) d(\pi) .
$$

Proof The proof depends on an elementary decomposition of the space of functions (see, for example, [17, p. 35]).

Let $V(b, k)$ and $U(b, k)$ be the modules generated by the injections $[b] \rightarrow[k]$ and the functions $[b] \rightarrow[k]$ respectively. A function $\sigma:[b] \rightarrow[k]$ takes exactly $r$ distinct values, for some $1 \leq r \leq b$, and so determines a partition of $[b]$ into $r$ parts. The set of all functions that induce a given partition generates a submodule isomorphic to $V(r, k)$. Hence, for $1 \leq r \leq b, U(b, k)$ has $S(b, r)$ submodules $V(r, k)$, and each of these has $e(r, \pi)$ constituents of dimension $m_{\pi}(k)$. (The constituents depend on $r$, but not the partition.) It follows from the decomposition of $V$ quoted above that $U(b, k)$ has

$$
\sum_{r=1}^{b} S(b, r) e(r, \pi)=\sum_{r=1}^{b} S(b, r)\left(\begin{array}{c}
r \\
|\pi|
\end{array}\right) d(\pi)
$$

constituents of dimension $m_{\pi}(k)$.

Our main result is now at hand. Given a permutation $\alpha \in \operatorname{Sym}_{k}$, let $R(\alpha)$ be the matrix representing the action of $\alpha$ on $U(b, k)$, that is,

$$
R(\alpha)_{\sigma \tau}= \begin{cases}1 & \text { if } \alpha \sigma=\tau \\ 0 & \text { otherwise }\end{cases}
$$

It is clear that the transfer matrix $M=M(k, y)$ satisfies $M R(\alpha)=R(\alpha) M$ for all $\alpha$ in Sym $_{k}$. In other words, $M$ belongs to the commutant algebra of the module $U(b, k)$. 
An important result [14, Theorem 1.7.8] tells us that the constituents of the commutant algebra correspond to those of the module, but with the dimensions and multiplicities switched. It follows that $M(k, y)$ has $m_{\pi}(k)$ constituents $M_{\pi}(k, y)$ for each $\pi$ with $|\pi| \leq b$, where $M_{\pi}(k, y)$ is a matrix of size $e^{*}(b, \pi)$.

When we substitute $k=(x-1)(y-1)$ in $M(k, y)$ and apply Theorems 1 and 2, powers of $y-1$ arise. In fact, for each constituent $M_{\pi}(k, y)$, we can write

$$
M_{\pi}((x-1)(y-1), y)=(y-1)^{b} N_{\pi}(x, y),
$$

where $N_{\pi}(x, y)$ is a matrix of size $e^{*}(b, \pi)$ with entries in $\mathbb{Z}[x, y]$.

Theorem 4 Let $\left\{G_{n}\right\}$ be a family of bracelets with the base graph having $b$ vertices. Then the Tutte polynomials of the graphs $G_{n}$ can be written in the form of a sum over all integer-partitions $\pi$ with $|\pi| \leq b$ :

$$
(x-1) T\left(G_{n} ; x, y\right)=\sum_{\pi} m_{\pi}(x, y) \operatorname{tr}\left(N_{\pi}(x, y)\right)^{n} .
$$

Here $m_{\pi}(x, y)$ is a polynomial function of $k=(x-1)(y-1)$, and $N_{\pi}(x, y)$ is a matrix of size $e^{*}(b, \pi)$ with entries in the ring $\mathbb{Z}[x, y]$.

For $b=2,3$, the dimensions of the relevant matrices are

$\begin{array}{cccccccc}\pi & {[0]} & {[1]} & {[20]} & {[11]} & {[300]} & {[210]} & {[111]} \\ d(\pi) & 1 & 1 & 1 & 1 & 1 & 2 & 1 \\ e^{*}(2, \pi) & 2 & 3 & 1 & 1 & - & - & - \\ e^{*}(3, \pi) & 5 & 10 & 6 & 6 & 1 & 2 & 1\end{array}$

\section{The case $|V|=2$}

It follows from the theory given above that, for any family of bracelets in which the base graph $B$ has vertex-set $V=\{1,2\}$, the matrix $M=M(k, y)$ has

1 constituent of dimension 2 ,

$k-1$ isomorphic constituents of dimension 3,

$k(k-3) / 2$ isomorphic constituents of dimension 1 ,

$(k-1)(k-2) / 2$ isomorphic constituents of dimension 1 .

The constituents are represented by matrices which depend on the linking set $L$, although their sizes 2, 3, 1, 1 and their multiplicities do not.

In order to illustrate Theorem 4 we shall work out the details for the cases $B=K_{2}, L=\{11,22\}$, and $B=K_{2}, L=\{11,12,22\}$, where the bracelets are the cubic and quartic plane ladders $\mathrm{CPL}_{n}$ and $\mathrm{QPL}_{n}$ respectively. The results have previously been obtained by other methods $[6,15]$, and more recently Chang and Shrock $[7,8]$ have used methods that differ from the ones used here only in notation and presentation. 
The theory of Specht modules provides standard bases for the various constituents, but we can use any bases that arise naturally. For example, for the two-dimensional constituent, we define vectors $\mathbf{u}, \mathbf{v}$ as follows:

$$
\mathbf{u}_{\sigma}=1 \text { for all } \sigma, \quad \mathbf{v}_{\sigma}= \begin{cases}1 & \text { if } \sigma_{1}=\sigma_{2}, \\ 0 & \text { otherwise. }\end{cases}
$$

Lemma 4.1 For $\mathrm{CPL}_{n}$, the action of $M$ on the vectors $\mathbf{u}, \mathbf{v}$ is given by

$$
\begin{aligned}
& M \mathbf{u}=(y+k-1)^{2} \mathbf{u}+(y+k-1)^{2}(y-1) \mathbf{v}, \\
& M \mathbf{v}=(2 y+k-2) \mathbf{u}+\left(y^{2}+y+k-2\right)(y-1) \mathbf{v} .
\end{aligned}
$$

Proof We have

$$
(M \mathbf{u})_{\sigma}=\sum_{\tau} M_{\sigma \tau} \mathbf{u}_{\tau}=\sum_{\tau} M_{\sigma \tau} .
$$

Given $\sigma$, there is one $\tau$ for which $\tau_{1}=\sigma_{1}$ and $\tau_{2}=\sigma_{2}$ both hold, $2(k-1) \tau$ 's for which exactly one of $\tau_{1}=\sigma_{1}, \tau_{2}=\sigma_{2}$ holds, and $(k-1)^{2} \tau$ 's for which neither equation holds. If $\sigma_{1}=\sigma_{2}$, these three cases give $M_{\sigma \tau}=y^{3}, y^{2}, y$, respectively, while if $\sigma_{1} \neq \sigma_{2}$, the values are $M_{\sigma \tau}=y^{2}, y, 1$, respectively. Hence

$$
\begin{aligned}
M \mathbf{u} & =y(y+k-1)^{2} \mathbf{v}+(y+k-1)^{2}(\mathbf{u}-\mathbf{v}) \\
& =(y+k-1)^{2} \mathbf{u}+(y+k-1)^{2}(y-1) \mathbf{v} .
\end{aligned}
$$

The equation for $M \mathbf{v}$ is obtained in a similar way.

Substituting $k=(x-1)(y-1)$ in the coefficients obtained above, we find

$$
\begin{aligned}
& y+k-1=x(y-1), \quad 2 y+k-2=(x+1)(y-1), \\
& y^{2}+y+k-2=(x+y+1)(y-1) .
\end{aligned}
$$

Thus if $\mathbf{v}^{*}=(y-1) \mathbf{v}$, the action of $M$ on the subspace $\left\langle\mathbf{u}, \mathbf{v}^{*}\right\rangle$ is given by the matrix $(y-1)^{2} N_{[0]}$, where

$$
N_{[0]}=\left(\begin{array}{cc}
x^{2} & x^{2} \\
x+1 & x+y+1
\end{array}\right) .
$$

Next, the three-dimensional constituent, which corresponds to the partition [1]. For each $i \in\{1,2, \ldots, k\}$, define vectors $\mathbf{r}^{i}, \mathbf{s}^{i}, \mathbf{t}^{i}$ as follows:

$$
\begin{aligned}
& \mathbf{r}_{\sigma}^{i}=\left\{\begin{array}{ll}
1 & \text { if } \sigma_{1}=i, \\
0 & \text { otherwise }
\end{array} \quad \mathbf{s}_{\sigma}^{i}= \begin{cases}1 & \text { if } \sigma_{2}=i, \\
0 & \text { otherwise }\end{cases} \right. \\
& \mathbf{t}_{\sigma}^{i}= \begin{cases}1 & \text { if } \sigma_{1}=\sigma_{2}=i, \\
0 & \text { otherwise }\end{cases}
\end{aligned}
$$

Lemma 4.2 For $\mathrm{CPL}_{n}$, the action of $M$ on the vectors $\mathbf{r}^{i}, \mathbf{s}^{i}, \mathbf{t}^{i}$ is given by $M \mathbf{r}^{i}=(y-1)(y+k-1) \mathbf{r}^{i}+(y-1)^{2}(y+k-1) \mathbf{t}^{i}+(y+k-1)(\mathbf{u}+(y-1) \mathbf{v})$, 


$$
\begin{aligned}
M \mathbf{s}^{i} & =(y-1)(y+k-1) \mathbf{s}^{i}+(y-1)^{2}(y+k-1) \mathbf{t}^{i}+(y+k-1)(\mathbf{u}+(y-1) \mathbf{v}), \\
M \mathbf{t}^{i} & =(y-1) \mathbf{r}^{i}+(y-1) \mathbf{s}^{i}+(y-1)^{2}(y+2) \mathbf{t}^{i}+\mathbf{u}+(y-1) \mathbf{v} .
\end{aligned}
$$

Proof For convenience, when $\tau_{1}=a$ and $\tau_{2}=b$, we write $\tau=(a b)$ and $M_{\sigma \tau}=$ $M(\sigma,(a b))$. With this notation we have

$$
\left(M \mathbf{r}^{i}\right)_{\sigma}=\sum_{j=1}^{k} M(\sigma,(i j))
$$

There are five cases for this sum of $k$ terms.

(1) If $\sigma_{1}=i, \sigma_{2} \neq i$, one term is $y^{2}$, and the remaining $k-1$ terms are $y$.

(2) If $\sigma_{1} \neq i, \sigma_{2}=i$, one term is $y$, and the rest are 1 .

(3) If $\sigma_{1}=\sigma_{2}=i$, one term is $y^{3}$, and the rest are $y^{2}$.

(4) If $\sigma_{1}=\sigma_{2} \neq i$ one term is $y^{2}$, and the rest are $y$.

(5) If $\sigma_{1}, \sigma_{2}$ and $i$ are all different, one term is $y$, and the rest are 1 .

Hence

$$
\begin{aligned}
M \mathbf{r}^{i}= & y(y+k-1)\left(\mathbf{r}^{i}-\mathbf{t}^{i}\right)+(y+k-1)\left(\mathbf{s}^{i}-\mathbf{t}^{i}\right)+y^{2}(y+k-1) \mathbf{t}^{i} \\
& +y(y+k-1)\left(\mathbf{v}-\mathbf{t}^{i}\right)+(y+k-1)\left(\mathbf{u}-\mathbf{v}-\mathbf{r}^{i}-\mathbf{s}^{i}+2 \mathbf{t}^{i}\right) .
\end{aligned}
$$

Rearranging, we get the equation in the statement of the lemma.

The equation for $M \mathbf{s}^{i}$ follows by symmetry. For $M \mathbf{t}^{i}$, we have

$$
\left(M \mathbf{t}^{i}\right)_{\sigma}=M(\sigma,(i i)) \text {. }
$$

The values of this term in the five cases listed above are, respectively: $y, y, y^{3}, y, 1$. Hence

$$
M \mathbf{t}^{i}=y\left(\mathbf{r}^{i}-\mathbf{t}^{i}\right)+y\left(\mathbf{s}^{i}-\mathbf{t}^{i}\right)+y^{3} \mathbf{t}^{i}+y\left(\mathbf{v}-\mathbf{t}^{i}\right)+\left(\mathbf{u}-\mathbf{v}-\mathbf{r}^{i}-\mathbf{s}^{i}+2 \mathbf{t}^{i}\right),
$$

which reduces to the stated equation.

Lemma 4.2 shows that, in the action of $M$ on $\mathbf{r}^{i}, \mathbf{s}^{i}$, and $\left(\mathbf{t}^{*}\right)^{i}=(y-1) \mathbf{t}^{i}$, the coefficients of those vectors are given by the matrix

$$
(y-1)\left(\begin{array}{ccc}
y+k-1 & 0 & (y+k-1) \\
0 & y+k-1 & (y+k-1) \\
y-1 & y-1 & (y-1)(y+2)
\end{array}\right) .
$$

Changing the basis by adding suitable multiples of $\mathbf{u}$ and $\mathbf{v}^{*}$, we obtain a subspace on which the action of $M$ is represented by the matrix given above. On substituting $k=(x-1)(y-1)$ we obtain $(y-1)^{2} N_{[1]}$, where

$$
N_{[1]}=\left(\begin{array}{ccc}
x & 0 & x \\
0 & x & x \\
1 & 1 & y+2
\end{array}\right) .
$$


Note that there are $k$ such representations, one for each value of $i$. However, their 'sum' is zero, and so there are only $k-1$ linearly independent ones, in accordance with the fact that $m_{[1]}(k)=k-1$.

The one-dimensional representations are derived from the action of $M$ on the vectors $\mathbf{e}^{i j}$, where

$$
\left(\mathbf{e}^{i j}\right)_{\sigma}=\left\{\begin{array}{ll}
1 & \text { if } \sigma_{1}=i, \sigma_{2}=j, \\
0 & \text { otherwise }
\end{array} \quad(i \neq j) .\right.
$$

A simple calculation gives the equation

$$
M \mathbf{e}^{i j}=(y-1)^{2} \mathbf{e}^{i j}+\text { terms in the vectors defined above. }
$$

This leads to $k(k-1)$ representations with matrix $\left[(y-1)^{2}\right]$. The representations corresponding to [20] and [11] are the same, and taking into account the linear dependencies, we obtain a total of

$$
m_{[20]}(k)+m_{[11]}(k)=k^{2}-3 k+1
$$

independent representations. Since $N_{[20]}$ and $N_{[11]}$ are both equal to the matrix [4], there is a 'constant' term $\delta(x, y)$, obtained by substituting $k=(x-1)(y-1)$ in $k^{2}-3 k+1$.

The Tutte polynomial for $\mathrm{CPL}_{n}$ is therefore

$$
(x-1) T\left(\mathrm{CPL}_{n} ; x, y\right)=\operatorname{tr}\left(N_{[0]}\right)^{n}+(x y-x-y) \operatorname{tr}\left(N_{[1]}\right)^{n}+\delta(x, y),
$$

where $N_{[0]}, N_{[1]}$ are the matrices displayed above, and

$$
\delta(x, y)=x^{2} y^{2}-2 x^{2} y-2 x y^{2}+x^{2}+x y+y^{2}+x+y-1 .
$$

For any bracelet with $b=2$, the decomposition of $M(k, y)$ takes the same form as for $\mathrm{CPL}_{n}$, and we can use the bases given above. For example, when $L=\{11,12,22\}$, we get the quartic plane ladders $\mathrm{QPL}_{n}$.

Lemma 4.3 For $\mathrm{QPL}_{n}$, the action of $M$ on the vectors $\mathbf{u}, \mathbf{v}, \mathbf{r}^{i}, \mathbf{s}^{i}, \mathbf{t}^{\mathbf{i}}$ is given by

$$
\begin{aligned}
M \mathbf{u}= & \left(2 y^{2}+(3 k-4) y+k^{2}-3 k+2\right) \mathbf{u}+(y-1)(y+k-1)\left(y^{2}+y+k-2\right) \mathbf{v}, \\
M \mathbf{v}= & \left(y^{2}+y+k-2\right) \mathbf{u}+(y-1)\left(y^{3}+y^{2}+k-2\right) \mathbf{v} ; \\
M \mathbf{r}^{i}= & (y-1)(2 y+k-2) \mathbf{r}^{i}+(y-1)^{2}\left(y^{2}+y+k-2\right) \mathbf{t}^{i} \\
& +(2 y+k-2) \mathbf{u}+(y-1)\left(y^{2}+y+k-2\right) \mathbf{v}, \\
M \mathbf{s}^{i}= & (y-1)(y+k-1) \mathbf{r}^{i}+(y-1)(y+k-1) \mathbf{s}^{i}+(y-1)^{2}(y+2)(y+k-1) \mathbf{t}^{i} \\
& +(y+k-1)(\mathbf{u}+(y-1)(y+k-1) \mathbf{v}), \\
M \mathbf{t}^{i}= & \left(y^{2}-1\right) \mathbf{r}^{i}+(y-1) \mathbf{s}^{i}+(y-1)^{2}\left(y^{2}-y+2\right) \mathbf{t}^{i}+\mathbf{u}+(y-1) \mathbf{v} .
\end{aligned}
$$

Applying the same reductions as for $\mathrm{CPL}_{n}$, the result is as follows. 
Theorem 5 The Tutte polynomials for the graphs $\mathrm{QPL}_{n}$ are given by

$$
(x-1) T\left(\mathrm{QPL}_{n} ; x, y\right)=\operatorname{tr}\left(N_{[0]}\right)^{n}+(x y-x-y) \operatorname{tr}\left(N_{[1]}\right)^{n}+\delta \text {, }
$$

where $N_{[0]}, N_{[1]}$, and $\delta$ are

$$
\begin{aligned}
& N_{[0]}=\left(\begin{array}{cc}
x+x^{2} & x(1+x+y) \\
1+x+y & 1+x+2 y+y^{2}
\end{array}\right), \quad N_{[1]}=\left(\begin{array}{ccc}
1+x & 0 & 1+x+y \\
x & x & 2 x+x y \\
1+y & 1 & 2+2 y+y^{2}
\end{array}\right), \\
& \delta=x^{2} y^{2}-2 x^{2} y-2 x y^{2}+x^{2}+x y+y^{2}+x+y-1 .
\end{aligned}
$$

\section{References}

1. Biggs, N.L.: Interaction Models. University Press, Cambridge (1977)

2. Biggs, N.L.: Chromatic polynomials and representations of the symmetric group. Linear Algebra Appl. 356, 3-26 (2002)

3. Biggs, N.L.: Specht modules and chromatic polynomials. J. Comb. Theory, Ser. B 92, 359-377 (2004)

4. Biggs, N.L., Damerell, R.M., Sands, D.A.: Recursive families of graphs. J. Comb. Theory, Ser. B 12, 123-131 (1972)

5. Brylawski, T., Oxley, J.G.: The Tutte polynomial and its applications. In: Matroid Applications. University Press, Cambridge (1992)

6. Chang, S.-C., Shrock, R.: Structural properties of Potts model partition functions and chromatic polynomials for lattice strips. Physica A 296, 131-182 (2001)

7. Chang, S.-C., Shrock, R.: Transfer matrices for the partition function of the Potts model on cyclic and Möbius lattice strips. Physica A 347, 314-352 (2005)

8. Chang, S.-C., Shrock, R.: Transfer matrices for the partition function of the Potts model on lattice strips with toroidal and Klein bottle boundary conditions. Physica A 364, 231-262 (2006)

9. Fortuin, C.M., Kasteleyn, P.W.: On the random cluster model. Physica 57, 536-574 (1972)

10. Kramers, H.A., Wannier, G.H.: Statistics of the two-dimensional ferromagnet I. Phys. Rev. 60, 252$262(1941)$

11. Kramers, H.A., Wannier, G.H.: Statistics of the two-dimensional ferromagnet II. Phys. Rev. 60, $263-$ $276(1941)$

12. Richard, J.-F., Jacobsen, J.L.: Character decomposition of Potts model partition functions II: toroidal geometry. Nucl. Phys. B 760, 229-249 (2006)

13. Royle, G.F., Sokal, A.D.: The Brown-Colbourn conjecture on zeros of reliability polynomials is false. J. Comb. Theory, Ser. B 91, 345-360 (2004)

14. Sagan, B.E.: The Symmetric Group. Springer, New York (2001)

15. Sands, D.A.: Dichromatic polynomials of linear graphs. Ph.D. thesis, University of London (1972)

16. Sokal, A.D.: The multivariate Tutte polynomial. In: Surveys in Combinatorics 2005, pp. 173-226. University Press, Cambridge (2005)

17. Stanley, R.P.: Enumerative Combinatorics I. University Press, Cambridge (1997)

18. Tanguy, C.: What is the probability of connecting two points? J. Phys. A, Math. Theor. 40, 14099$14116(2007)$

19. Temperley, H.N.V., Lieb, E.H.: Relations between the 'percolation' and 'colouring' problem and other graph-theoretical problems associated with regular planar lattices: some exact results for the 'percolation' problem. Proc. R. Soc. Lond., Ser. A 322, 251-280 (1971)

20. Welsh, D.J.A.: Complexity: Knots, Colourings, Counting. LMS Lecture Notes Series, vol. 186. University Press, Cambridge (1993) 